\title{
Aproximaciones al desarrollo territorial desde un análisis del periurbano en Boyacá, Colombia
}

\author{
APPROACH TO TERRITORIAL DEVELOPMENT FROM ANALYSIS \\ OF PERIURBAN IN BOYACÁ, COLOMBIA
} APROXIMAC̣ÕES AO AL DESENVOLVIMENTO TERRITORIAL DESDE
UMA ANÁLISE DO PERIURBANO EM BOYACÁ, COLÔMBIA

DANILO CASTRO Ricaurte

Evaluación:

24 de julio de 2017

Geográfica, 22(2),123-136. doi: 10.19053/01233769.5956

Aprobación:

13 de agosto de 2017

\section{Resumen}

La zona central constituye el territorio con mayor desarrollo, calidad de vida y actividades socioeconómicas del departamento de Boyacá, la cual se extiende a lo largo del valle del río Chicamocha. Esta región tiende a integrarse gracias a las condiciones geográficas, usos y aprovechamientos del territorio a partir de una red de municipios que tiene como eje a Tunja, Duitama y Sogamoso, red que favorece la movilidad de la población y la diversidad de actividades en los municipios de la región. El presente artículo busca aportar al desarrollo regional a partir del análisis del periurbano de Tunja, Duitama, Sogamoso. El trabajo abarcó tres etapas: análisis de literatura y conceptos, trabajo de campo (para esto se realizó la aplicación de encuestas a la población y entrevistas a funcionarios de los municipios) y la formulación de un modelo de desarrollo

1 Ingeniero geógrafo y ambiental de la Universidad de Ciencias Aplicadas y Ambientales (UDCA). Maestrando en Estudios Urbano Regionales de la Universidad Nacional de Colombia, Sede Medellín. dacastrori@unal.edu.co 
territorial con enfoque regional y no solo urbano, como muchas propuestas actuales en Boyacá y Colombia.

Palabras clave: desarrollo regional, modelo, municipio, periurbano, región, territorio.

\section{Abstract}

Boyacá's central region is the area with the highest development, quality of life and socio-economic activities of the department which extends along the valley of the Chicamocha River. This region tends to integrate through to the geographical conditions, use and exploitation of the territory from a network of municipalities whose axis to Tunja, Duitama and Sogamoso, which encourages the mobility of the population and diversity of activities in the municipalities in the region. The following paper seeks to contribute to regional development through the analysis of periurban Tunja, Duitama, Sogamoso. The work comprised three stages: First, analysis of literature and concepts; Second, fieldwork (for implementing this population surveys and interviews with municipal officials was held); and third, the development of a territorial development model with regional focus and not only urban development as many current proposals in Boyacá and Colombia.

Keywords: model, municipality, periurban territory, region, regional planning.

\section{Resumo}

A zona central constitui o território com maior desenvolvimento, qualidade vida e atividades socioeconómicas do departamento de Boyacá, que se estende pelo vale do Río Chicamocha. Essa região tende a se integrar pelas condições geográficas, usos e aproveitamentos do território a partir de uma rede de municípios que tem como eixo a Tunja, Duitama e Sogamoso, essa rede favorece a mobilidade da população e a diversidade de atividades nos municípios da região. $\mathrm{O}$ artigo busca aportar ao desenvolvimento regional a partir da análise do periurbano de Tunja, Duitama, Sogamoso. O trabalho abarcou três etapas: análise de literatura e conceitos, trabalho de campo (para isso foi feita a aplicação de enquetes à população e entrevistas aos funcionários dos municípios) e a formulação de um modelo de desenvolvimento territorial com enfoque regional e não simplesmente urbano como muitas propostas atuais em Boyacá e Colômbia.

Palavras chave: desenvolvimento regional, modelo, município, periurbano, região, território. 


\section{Introducción}

Los grupos poblacionales buscan constantemente la mejor forma de relacionarse en el territorio que habitan y la forma e intensidad como aprovechan los recursos disponibles en este, con el fin de elevar su calidad de vida y disfrutar un ambiente sustentable. Para llevar a cabo estos planes, se formulan propuestas relacionadas con la forma de administrar el territorio, su planificación y gestión con el fin de generar una visión de desarrollo que beneficie a la población. Esa visión se construye a partir de la forma como se aprovechan los recursos disponibles y las capacidades de la población para elevar la calidad de vida de todos.

Hay diferentes formas de concebir el territorio: por un lado, como una construcción de lazos sociales a partir de la interacción y cooperación de los individuos (Fligstein, 2001); o como un elemento físico donde se asienta la población y tienen lugar las dinámicas económicas, sociales, culturales y políticas de la sociedad (Departamento Nacional de Planeación-DNP, 2009). A pesar que el desarrollo es un proceso esencialmente humano, se da sobre territorios concretos (Programa de las Naciones Unidas para el Desarrollo-PNUD, 2002). Este ayuda a forjar identidades, rasgos culturales y relaciones socioeconómicas, las cuales dotan de características particulares al territorio, al tiempo que determinan el nivel de desarrollo de las comunidades allí asentadas.

De la unión de estos dos conceptos surge un proceso de cambio llamado desarrollo territorial (DNP, 2009). Este aboga por el mejoramiento de la calidad de vida a partir de propiciar la armonía entre población, uso del territorio, conservación y protección de los recursos (DNP, 2010). Se debe basar en una estrategia clave para la descentralización de los sistemas decisionales
(Boisier, 1998), definitivos para la consolidación de procesos de concertación local (Pichierri, 2002), que permitan la formulación de modelos concertados de desarrollo territorial de largo plazo y que involucren la intervención pública y privada, en el desarrollo local, regional y nacional (Porras, 2006).

Sin embargo, el crecimiento de las ciudades, la especialización de sus funciones, la multiplicidad de los servicios prestados, en gran parte gracias al avance de los medios de comunicación e información, y el progreso de la infraestructura disponible han contribuido a eliminar drásticamente las diferencias en la relación campo-ciudad, especialmente en aquellos territorios próximos a grandes centros urbanos que se han beneficiado de los avances descritos anteriormente; antes, solían diferenciarse claramente los componentes rurales de los urbanos y la población que habitaba en cada uno de estos territorios. Estas características generan un consenso más que un énfasis en las diferencias entre rural y urbano (Allen, 2003).

A finales del siglo XX surge el concepto de periurbano, definido por el Instituto de Estudios Geográficos de Francia (IEG, 1994 como se cita en Navarro, 2006) como "el espacio situado alrededor de las ciudades, susceptible a su influencia directa y de ser significativamente tocado por los procesos puestos en marcha por esa proximidad" (p. 249). Salazar (2010) lo explica como la periferia de la ciudad aún sin urbanizar, carente de infraestructura urbana y servicios públicos o albergándola con deficiencias, con especial potencial para el asentamiento de nueva población, aglomeraciones urbanas y actividades económicas empujadas del centro a la periferia en gran medida por la influencia de los precios del suelo.

Si bien es indudable que hay elementos de ruralidad paisajísticos y de laboreo contenidos en este espacio, los actores de la producción devienen, 
esencialmente, en actores urbanos. Allen (2003) añade que si bien el periurbano "está sujeto constantemente a cambios y transformaciones, la mayoría de dichos cambios son impulsados por la proximidad de áreas urbanas" (p. 1). Sin embargo, estos cambios afectan la sustentabilidad de los recursos naturales y la calidad de vida de las personas.

Por otro lado, la sustentabilidad del aprovechamiento de recursos naturales depende del traslado de los costos ambientales de zonas rurales y urbanas al periurbano, ya que esta última tiende a poseer características de las otras y soportar las presiones por la diversificación de actividades socioeconómicas y búsqueda de espacio productivo para vivienda campestre, traslado de actividades industriales desde las ciudades, cultivos transitorios de esencia agroindustrial, entre otras.

Estas características describen la situación que se viene presentando en el territorio central de Boyacá, cuyos municipios principales — Tunja, Duitama, Sogamoso - han venido presentando un crecimiento urbano iniciado con una urbanización en zonas alejadas de los cascos urbanos, intercalando zonas sin urbanizar que con el tiempo terminan siendo urbanizadas, aumentando el tamaño del área urbana (Herrera, 2009). Sin embargo, diversos factores socioeconómicos y características de la población han incrementado las relaciones entre los diferentes municipios de la región, sobrepasando la capacidad de acción de los administradores locales ${ }^{2}$ ya que las decisiones tomadas empiezan a impactar en el

2 Según Borja y Castells (1998), en el nuevo sistema global los Estados-nación son demasiado pequeños para controlar y dirigir los flujos globales de poder, riqueza y tecnología; pero son muy grandes para representar la pluralidad de los intereses sociales e identidades culturales de la sociedad. ámbito regional, demandando nuevas formas de relación entre los municipios y la población.

Por otra parte, las iniciativas previas de asociatividad planteadas para la zona de estudio priorizan la urbanización y proponen escenarios de metropolización que desconocen particularidades inherentes tanto a la región como su población. Estas propuestas vienen formuladas desde el DNP (2011), gobernación de Boyacá y alcaldías municipales (región económica de planificación y corredor estratégico de Boyacá).

Es por esto que la formulación de una visión de desarrollo territorial aplicada a una región común (DNP, 2010) surge como una prioridad a partir de la profundización de las relaciones entre los habitantes de los municipios del centro de Boyacá, cuya interacción se ve reflejada en las diferentes actividades llevadas a cabo en especial sobre el periurbano regional, que permite tal interacción social, económica y ambiental. Este tipo de propuestas deben servir de ejemplo para su aplicación en otras regiones, partiendo de la implementación en territorios intermedios, y no en aquellos que concentran más desarrollo y población en el país, en aras de cumplir con los objetivos de desarrollo del milenio (PNUD, 2012) planteados para Boyacá y el país.

\section{Metodología de trabajo}

La metodología para desarrollar el proyecto se llevó a cabo en tres etapas: la primera consistió en una revisión de bibliografía, recolección de imágenes satelitales, cartografía y documentos referentes a la zona de estudio. La segunda etapa consistió en trabajo de campo dividido en tres partes: aplicación de 10 entrevistas a funcionarios de los municipios, 
FIGURA 1.

Localización zona de estudio

Fuente: Elaboración propia.
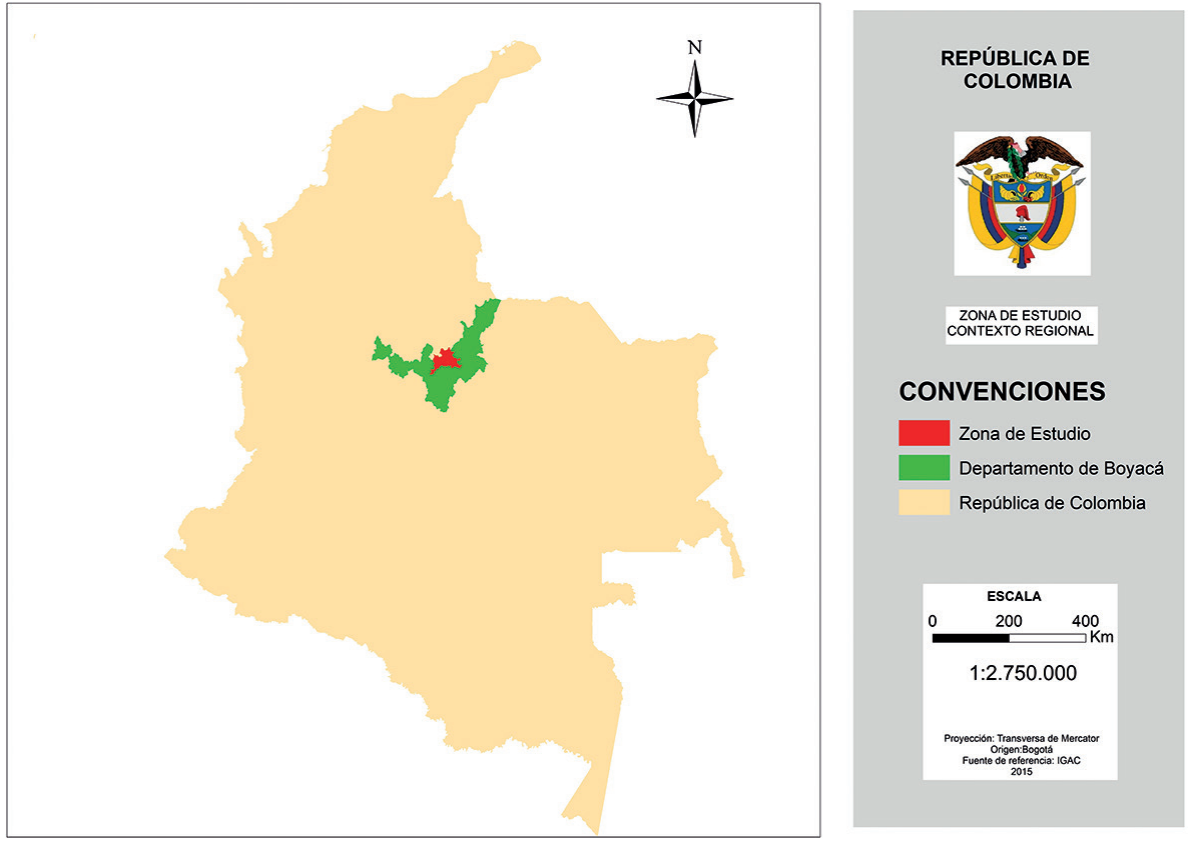

aplicación de 123 encuestas a la población y observación directa del territorio de estudio. La tercera etapa consistió en la formulación de la propuesta de un modelo de desarrollo territorial para la zona de estudio. Dicha propuesta fue resultado del análisis de los resultados obtenidos en las etapas previas.

\subsection{Delimitación de la zona de estudio}

Para el desarrollo del proyecto, se partió del análisis de los municipios Tunja, Duitama y Sogamoso, ya que son los principales centros urbanos no solo de la región sino del departamento de Boyacá. Entre estos se ubican una serie de municipios (Figura 1) con los cuales comparten dinámicas poblacionales, actividades productivas, prestación de servicios educativos, entre otros. También comparten jurisdicción sobre el valle del río Chicamocha y son atravesados por la doble calzada Bogotá-Sogamoso, la cual no solo es el eje vial principal, sino también de desarrollo y crecimiento urbano.

A lo largo de la doble calzada, se constituye el Corredor Industrial de Boyacá, cuyas actividades empiezan a ser notorias desde la planta de DIACO en el municipio de Tuta, pasando por un incremento de las actividades industriales entre Paipa y Duitama, hasta llegar a Sogamoso-Nobsa, donde hay emplazamiento de industria pesada. Esto permite identificar dos grandes zonas en la región de estudio (Figura 2); una primera correspondiente a Tunja y su zona de influencia, que se destaca por una alta concentración de la población urbana en Tunja y alrededores. La segunda zona está comprendida entre Paipa y Sogamoso, abarcando casi toda la actividad industrial emplazada en el valle del río Chicamocha; esta región la denominaremos "Valle Central de Boyacá", debido a las características anteriormente descritas. 


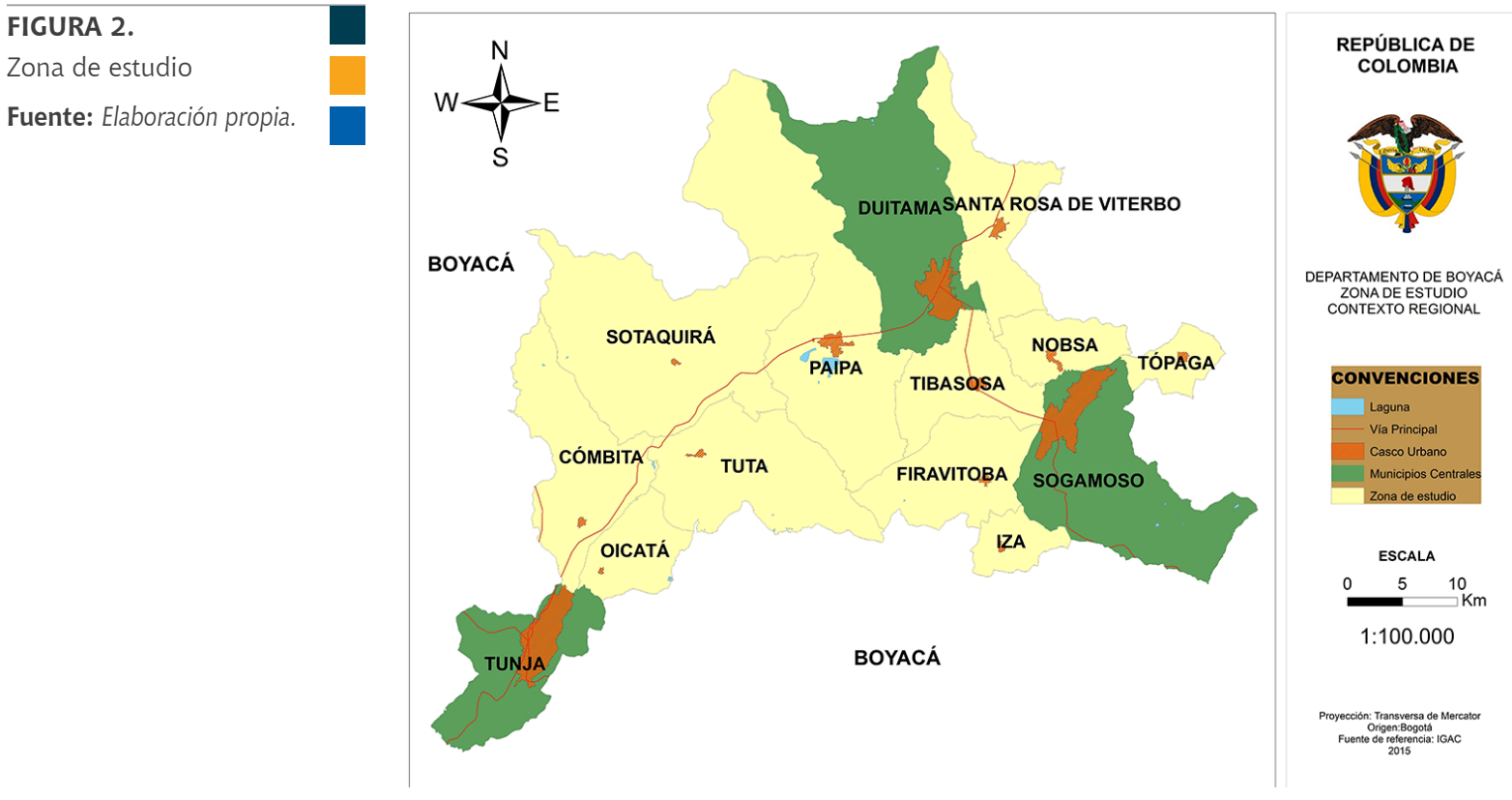

\section{Análisis y resultados}

A pesar de que la formulación de la Ley 388 de 1997 buscaba establecer un marco normativo que orientara las formas de uso del suelo, desde las alcaldías municipales se percibe la falta de coordinación para adelantar proyectos, gestionar recursos y definir una visión de territorio clara en el plan de desarrollo y los Planes de Ordenamiento Territorial (en adelante POT). Como menciona Carvajal (2011), esta Ley impone un horizonte de actuación en el cual "orienta" la forma en la que se debe ver al municipio, obligándolo a cumplir con la normatividad en el marco de una autonomía guiada. Sin embargo, aquello se dificulta a raíz de las obligaciones que deben asumir las administraciones municipales. A pesar de esto, cabe resaltar que la Ley 1454 de 2011 (Ley Orgánica de Ordenamiento Territorial, en adelante LOOT) aporta importantes elementos para desarrollar los postulados de la Ley 388 por medio de esquemas asociativos, específicamente el artículo 16, el cual permite la creación de pro- vincias administrativas de planificación, a partir de las intenciones de dos o más municipios, con el fin de sacar adelante proyectos de desarrollo integral.

Desafortunadamente, estas ideas aún son incipientes y demandan un fuerte apoyo y cooperación entre administraciones municipales, instituciones y población, ya que sería un gran avance consolidar una política común respecto a la forma de regular el uso y aprovechamiento de los territorios periurbanos, que en últimas son donde se evidencia el avance de los procesos de integración social, económico $\mathrm{y}$ ambiental a nivel regional.

Esta afirmación tiene sustento en datos recolectados en la Gobernación de Boyacá, desde la cual se observa falta de iniciativa a nivel municipal sumado a una baja capacidad de gestión y administración territorial. Estas falencias (ligadas a la forma de actuación de las instituciones) pueden encontrar correctivo por medio de la asociatividad regional, permitiendo el desarrollo de mejores prácticas administrativas que permitan el surgimiento de iniciativas que no limiten 
su actuación a un solo municipio, sino que sirva para el beneficio de toda la región, en especial en los temas destacados por la LOOT (2011) en el artículo 16 (prestación de servicios públicos, ejecución de obras de ámbito regional, ejecución de proyectos de desarrollo integral y gestión ambiental).

\subsection{Análisis de resultados trabajo de campo}

A partir de los recorridos efectuados en la zona de estudio, se observa una complementariedad en las actividades socioeconómicas de la población definidas para cada núcleo, por ejemplo, Tunja se destaca como centro administrativo, de servicios y de educación superior. Por su parte, la dinámica industrial prima en Duitama y Sogamoso. Esta última tiene un claro enfoque hacia la elaboración de materias primas para la construcción (cemento y acero). Sin embargo, las actividades industriales se mezclan con actividades agropecuarias en la zona rural del valle del Chicamocha.

Debido a la homogeneidad geográfica y de actividades socioeconómicas, la región se resalta en su conjunto y no cada municipio individualmente. Un ejemplo es el turismo, el cual se destaca por la variedad y oferta en toda la región, abarcando varios campos (turismo ecológico, cultural, de salud, variedad gastronómica y de paisajes). Reconocer estas características para pensar el territorio es fundamental en el planteamiento del desarrollo regional, con el fin de pensar en conjunto lo urbano y lo rural. Para ello, profundizar conceptos como periurbano ha de servir para identificar las actividades y usos en zonas urbanas, rurales y suburbanas, donde rural y suburbano no sean vistos solo como zonas de incorporación urbana.

\subsection{Análisis de la encuesta aplicada a la población}

La encuesta aplicada a la población se estructuró en tres partes para conocer la opinión de esta respecto a diferentes aspectos del territorio donde viven. La primera sección de preguntas buscó la contextualización de la zona a partir de la visión de sus habitantes, los usos o aprovechamientos llevados a cabo allí y características (físicas, geográficas, sociales, culturales) propias de la zona de estudio. La segunda parte indagó sobre el concepto de periurbano y la clasificación de usos del suelo incluidos en los planes de ordenamiento territorial POT de los municipios. El tercer bloque de la encuesta preguntó sobre el manejo dado desde la administración municipal a la zona, los proyectos relacionados y la forma como se gestiona no solo el suelo sino el territorio.

En la primera parte, los encuestados concuerdan en que prefieren vivir en zona rural, debido a que los servicios públicos son más económicos y desarrollan algún tipo de actividad económica. Adicionalmente, notan un cambio en la urbanización de la zona, debido principalmente a la construcción de la doble calzada, que ha provocado un incremento de la construcción de vivienda, locales comerciales, emplazamiento de fábricas y estaciones de servicio. La existencia de estos nuevos equipamientos hace que la población residente demande la construcción de equipamientos de salud, educación, recreación y administrativos con el fin de evitar ir hasta los centros urbanos ya que ellos consideran que estos se podrían prestar más cerca y reducir así los trayectos de desplazamiento. También serviría para el disfrute de la población de paso que llega y los turistas, quienes verían con agrado la prestación de servicios tradicionalmente ofrecidos en cascos urbanos. 
En la segunda parte, los encuestados no relacionan su territorio con conceptos como periurbano o suburbano, ya que generalmente lo asocian como rural, pero en zonas cercanas a Paipa y Duitama, la mezcla de actividades es llamativa. Hay industrias de metalurgia y metalmecánica, vivienda residencial, cultivos y parques industriales. Esta mezcla de actividades otorga características urbanas a las zonas cercanas al corredor vial, predominando las rurales a medida que se aleja de este.

Las respuestas de la tercera parte muestran dos visiones: una de abandono por parte de la administración de los municipios cuyos intereses desconocen el potencial de uso y aprovechamiento del territorio. La otra visión se relaciona con el anhelo de la gente por conservar los rasgos que identifican a la zona que habitan. La población no expresa un deseo de abandonar su territorio ya que la mayoría se encuentra a gusto en este, pero la falta de oportunidades laborales y sociales obliga a que tengan que ir a la ciudad a suplir estas carencias. Además, prefieren que las oportunidades laborales se den en actividades ligadas al territorio y su uso tradicional.

A pesar de considerarse como un hecho la urbanización a lo largo del corredor vial, esta puede generar opciones de ocupación debido a los cambios socioeconómicos generados por la mayor dinámica de este. En las zonas agropecuarias que hacen parte del distrito de riego del Alto Chicamocha se prevé un fortalecimiento debido a la productividad del suelo, si hay una buena administración del distrito de riego que promueva la tecnificación de la producción agropecuaria orientada a la agroindustria y cultivos orgánicos. Sin embargo, se espera un rezago en las zonas rurales que no hacen parte del distrito de riego, continuando con las actividades agropecuarias de subsistencia sin generar bienestar y desarrollo para sus habitantes.

\section{Propuesta de un modelo de desarrollo territorial}

En la formulación de la propuesta de modelo de desarrollo regional debemos incluir algunos elementos teóricos. Abramovay (2006) menciona que los "procesos de cooperación entre municipios pueden ser un medio para atenuar la propensión que tienen las organizaciones territoriales de adquirir, en la práctica, un carácter sectorial" (p. 53). Esto implica que en la voluntad colectiva radica la clave para generar cambios y enfocar la atención respecto a administrar de una mejor manera y en conjunto los recursos de que se dispone en el territorio habitado.

A su vez, indica que el territorio enfatiza la manera como una sociedad utiliza los recursos de los que dispone en su organización productiva y, por lo tanto, en la relación entre sistemas sociales y ecológicos (Abramovay, 2006). Es decir, los recursos disponibles y las condiciones del territorio condicionan las formas de interacción de la población con este, y la variedad y tipo de actividades que se pueden desarrollar.

Vásquez (2009) afirma que el desarrollo local comparte objetivos con las políticas estructurales de un país. Sin embargo, difiere con esta ya que el desarrollo local define sus acciones con un enfoque territorial. Dicho de otro modo, el objeto del desarrollo local es potenciar las capacidades y fortalezas del territorio y su población, lo cual permite mejorar la competitividad y la productividad territorial para fortalecer y diversificar las actividades socioeconómicas y potenciar la inversión en infraestructura para la mejora de la calidad de vida y ambiente de la población y el territorio. Aunque Bervejillo (1996) plantea un escenario complejo debido a la influencia de la globalización y su impacto en la diversidad cultural y las identidades sociales. 
Para ello, la toma de decisiones debe contar con la participación de actores locales con el fin de garantizar el cumplimiento de los objetivos comunes que atañe a la construcción de tejido social y redes de cooperación territorial, por medio de la asociación de las comunidades, haciendo parte de la denominada economía social. Mozas y Bernal (2006) destacan las entidades de economía social por la labor social que prestan, más allá del alcance de las entidades administrativas. Al asociarse las comunidades, se pueden generar ideas colectivas que sienten bases respecto a la visión que se tiene del territorio, su potencial de aprovechamiento y lo que se espera de este. Al respecto, es conveniente leer a Sabatini (1990), quien invita a investigar desde el empirismo de lo local las formas de desarrollo que permitan a las regiones elevar su calidad de vida.

A partir de las estrategias formuladas a nivel local, y su articulación con las propuestas de escala regional, se propone que los municipios de estudio a pesar de tener el potencial de consolidarse como centralidad para otros municipios del departamento de Boyacá, deben empezar a tomar decisiones en conjunto que permitan destacar y proyectarse como una región común y no como municipios independientes. Este carácter de región les va a permitir incrementar sus relaciones incluso con municipios de departamentos vecinos. Esta área de influencia incluye especialmente el sur de Santander, norte de Cundinamarca y Casanare, con los que mantiene estrechos lazos sociales, culturales y económicos.

Con base en lo anterior, es pertinente pensar el modelo regional no solo a partir de un beneficio para la población local, sino también para aquella que tiene relación con la región. La mirada del periurbano es significativa, ya que permite acotar el enfoque de región en tanto que abre la oportunidad de analizar las mixturas urbano-rurales y las dinámicas propias de la funcionalidad regional, ya que son las que otorgan un acento particular a la región. Su análisis debe ser el punto de partida para la construcción de políticas sectoriales comunes entre los municipios, propendiendo por una relación armónica entre la población y el territorio.

De esta forma, el modelo de desarrollo territorial que aquí se propone (Figura 3 ) debe trascender el debate de la urbanización y centrarse en las estrategias de acondicionamiento social y ambiental que permitan un desarrollo económico acorde al potencial regional que se sustenta en la oferta de recursos sociales y ambientales.

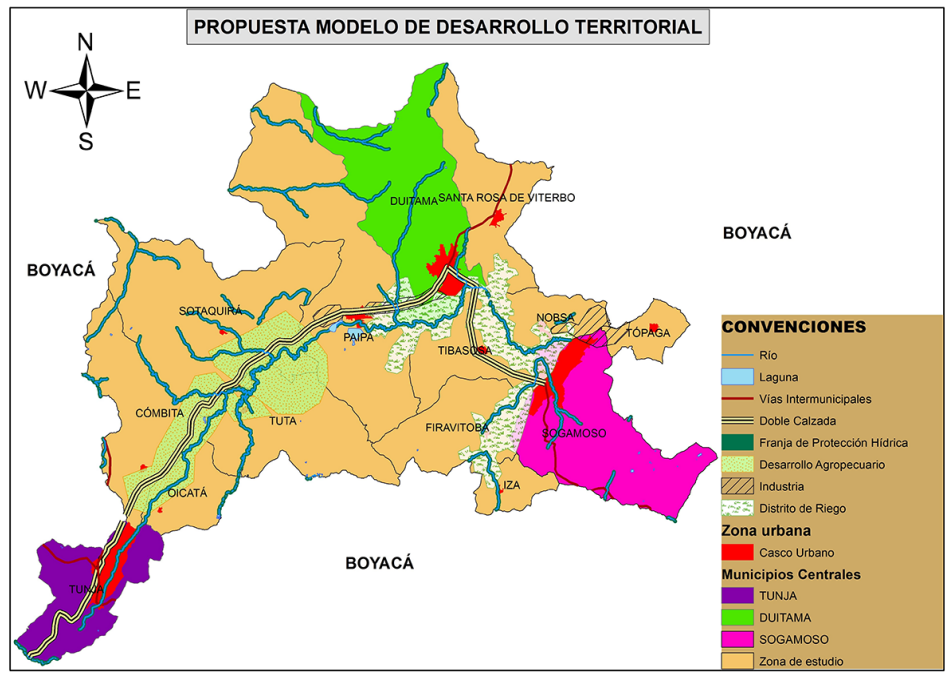

\section{FIGURA 3.}

Propuesta de modelo de desarrollo territorial

Fuente: Elaboración propia. 


\subsection{Estrategias de escala regional}

Hay aspectos generales que afectan directamente a la población de la región y cuya existencia es vital para potenciar o limitar el desarrollo regional. De esta manera, se identificaron los siguientes puntos, con el fin de promover una política regional que permita la equiparación en cada municipio de la región.

\subsubsection{Suministro de agua potable}

Es fundamental debido a que es el servicio público básico para la vida. Su oferta condiciona el crecimiento de la población y la orientación de su uso. Se debe lograr una oferta y suministro de agua potable igualitaria para la población sin importar a qué municipio pertenezca, a partir de la integración de acueductos, basados en el intercambio de experiencias a nivel urbano y rural. El trabajo conjunto es básico para consolidar propuestas que beneficien a toda la región.

\subsubsection{Tratamiento de aguas residuales y recu- peración de cuencas hidrográficas}

El eje integrador de la región es la cuenca del río Chicamocha, que inicia en Tunja con el río Jordán el cual se encuentra bastante deteriorado. Recuperar este afluente es vital para el mejoramiento de la calidad ambiental de Tunja, ya que es atravesada de sur a norte por este río. Es viable la creación de un parque ambiental alrededor de su curso, aprovechando que su ribera no está completamente urbanizada. Esta política de cuencas ha de servir como ejemplo para la recuperación y preservación de afluentes de otros municipios de la región con el fin de formular y seguir una política pública común que genere certezas respecto a la administración pública regional. El manejo adecuado del recurso hídrico disponible es importante para definir los distintos aprovechamientos que se pueden realizar, el cual debe ser complementado por una fuerte educación y conciencia ambiental en la población de toda la región.

\subsubsection{Infraestructura vial}

La doble calzada Bogotá-Sogamoso es el eje vial más importante de la región, ya que se constituye como el eje de crecimiento socioeconómico y de integración regional. Las demás vías de la región y aquellas que conectan con otras regiones se articulan a esta, asemejando una gran arteria (vial) a la que se unen pequeños conectores (demás vías intermunicipales). Este no solo ha disminuido la distancia, sino que ha incrementado las relaciones entre la población de la región. Su fortalecimiento es fundamental para la integración regional y el aumento productivo.

\subsubsection{Transporte}

El transporte permite a la población desplazarse entre municipios, facilitando trabajar en un municipio diferente al de residencia. Es fundamental para facilitar los intercambios de población, acceso a servicios y comunicación. La oferta de transporte - que vaya acompañada de calidad - debe estar orientada a facilitar la comunicación de la población, y su interacción con el territorio, de otra forma, serían esfuerzos vanos tratando de consolidar una visión regional sin facilitar alternativas de transporte a la población. Hay que fomentar la implementación de sistemas integrados de transporte en las tres ciudades principales. Debe desarrollarse mejorando la oferta y servicio de transporte público, al mismo tiempo que las empresas regulan la prestación del servicio, ya que, finalmente, este es prestado por empresas privadas pero que favorece a toda la población. 
Lograr el establecimiento de sistemas integrados de transporte en ciudades intermedias como las nuestras sería un logro destacable a nivel nacional que puede servir de ejemplo para otras regiones del país, teniendo en cuenta los casos no tan exitosos de sistemas masivos implementados en ciudades más grandes.

El manejo de las basuras debe seguir la lógica territorial que hasta el momento se ha planteado. Para Boyacá se tiene dos grandes rellenos sanitarios, localizados en Tunja y Sogamoso, y sirven a gran parte de los municipios. Sin embargo, falta una articulación entre las empresas prestadoras del servicio y los administradores de los rellenos sanitarios, pareciendo que la responsabilidad de aquellos que llevan sus basuras termina en el momento que las depositan en dichos rellenos.

Un manejo acertado debe repartir responsabilidades y generar apropiación entre la población de todos los municipios, debido a que una falla repercute en la calidad del servicio y la recolección de los desechos de estos. Fortalecer el reciclaje, la reutilización y alternativas de uso a los residuos sólidos para que sean incorporados a la cadena productiva, generando nuevas oportunidades de negocio y trabajo para la gente.

El aprovechamiento socioeconómico para el sector agropecuario identifica tres áreas con vocación diversa. Una primera ubicada entre Tunja y Paipa, donde se desarrollan varias actividades agropecuarias, pero con un especial acento en la producción frutícola. La segunda corresponde al distrito de riego Usochicamocha, orientado al cultivo de alimentos y producción lechera. La tercera área corresponde a las montañas que rodean el valle del río Chicamocha. Estas se encuentran subutilizadas y deterioradas, sin embargo, hay ejemplos de aprovechamientos en estas (viñedos en el sector Punta Larga) mostrando que una opción viable es la plantación de frutales y especies forestales, orientados a la manufactura agroindustrial. Esta actividad ayudaría a generar empleo, incrementar la productividad rural, diversificar las actividades urbanas y rurales así como disminuir tanto la presión del crecimiento urbano en los bordes de ciudad como la afectación y destrucción de ecosistemas sensibles y estratégicos.

A pesar de la existencia de industria pesada en la región (siderurgia y construcción), esta se ubica especialmente en Sogamoso, debido a la disposición de materias primas y condiciones geográficas para el establecimiento de factorías. En los últimos años se ha visto una mejora en la calidad del aire de esta zona, por lo tanto reglas claras para el desarrollo de la actividad industrial y el mejoramiento de los procesos productivos son aspectos importantes para acabar los estigmas generados alrededor de estos.

Para que tales condiciones favorables puedan lograrse, las empresas deben trabajar en conjunto con las administraciones locales y la población con el fin de definir los proyectos a ejecutar y los programas a desarrollar que aseguren la sustentabilidad ambiental de la región y generen beneficios para la población.

El turismo, que se viene consolidando fuertemente en la región, debe servir para integrar esas variedades de actividades expresadas por la población, de tal forma que se llegue a formar un clúster alrededor de esta actividad, que sirva para orientar la actividad regional al tiempo que se diferencie, con el objetivo de mostrar su variedad a partir de la construcción colectiva de visiones del territorio. Este tema es fundamental, ya que el turismo tiene el potencial de ser vitrina para la región y servir como canal para distribuir múltiples productos y servicios no solo a los turistas, sino también para crear alianzas 
y establecer redes que permitan a Boyacá opciones para insertarse en las economías de otras regiones y especialmente otros países. En últimas, es un sector que puede consolidarse como la base económica para la región y el departamento siempre y cuando se sepa aprovechar para diversificar las actividades y no generar una dependencia alrededor de este.

\section{Conclusiones}

La formulación de propuestas de desarrollo territorial sirve para dar respuesta a las necesidades de la población. Además, deben incluirla y abarcar el territorio que habitan. De esta manera, no se debe pensar un modelo individual para cada municipio, sino uno común para los municipios que comparten un mismo espacio geográfico. Esto puede permitir a futuro reducir costos de administración para liberar recursos que pueden utilizarse para inversión, investigación y educación de la población. La posibilidad de inversión conjunta en proyectos entre municipios es importante para evitar la atomización de recursos y la competencia entre estos, ya que no se traducen en beneficios para la población, sin embargo, el compartir experiencias sí sirve para ayudar a municipios menos desarrollados e impulsar planes de trabajo en pro del beneficio de toda la población.

En la zona de estudio se han podido identificar parte de estas características. Por parte de la población se notan aspectos que permiten identificar rasgos comunes independientes del municipio al que pertenezcan (especialmente en las actividades socioeconómicas). Desde las administraciones municipales también se observan progresos en la construcción de visiones comunes para la región, sin embargo, los municipios pequeños se ven un poco relegados de estas discusiones. A pesar de esto, conviene aclarar que los intentos de construcción de visiones regionales de desarrollo en Boyacá hasta ahora empiezan a ser un tema relevante en la forma de administrar el territorio, y tienen a su favor la posibilidad de integrar a todos los actores sociales para que sea un proceso exitoso.

Esto se ve influenciado por los cambios sobre la percepción y construcción del territorio para disminuir los desequilibrios en el desarrollo de las regiones en Colombia. Además, contribuye al descubrimiento propio del territorio, de las necesidades de la población y de lo que desean plantear como estrategia para el desarrollo del territorio, de manera que redunde en una mejora de la calidad de vida y contribuya a disminuir la presión sobre las regiones más desarrolladas y orientar de una forma racional el crecimiento urbano. Esta idea se relaciona con la importancia que ha venido cobrando el turismo en la región, las opciones y oportunidades que presenta para la población, donde la experiencia local basada en una oferta diferenciada en los municipios, contribuya a la vez a la construcción de una visión regional por parte de la población. 


\section{Referencias}

Abramovay, R. (2006). Para una teoría de los estudios territoriales. En M. Manzanal, M. Lattuada, y G. Neiman (Comps.), Desarrollo rural: organizaciones, instituciones y territorio (pp. 51-70). Buenos Aires: Ediciones CICCUS.

Allen, A. (2003). La interfase periurbana como escenario de cambio y acción hacia la sustentabilidad del desarrollo. Cuadernos del Cendes, 20(53), 7-21. Recuperado de http://www.scielo.org.ve/scielo.php?script=sci_arttext\&pid=\$1012$25082003000200002 \& \operatorname{lng}=e s \& n r m=$ iso

Bailly, A. (2009). Pour un développement urbain durable. Revue d’Economie Régionale et Urbaine, 2, 231-238.

Bervejillo, F. (1996). Territorios en la globalización cambio global y estrategias de desarrollo territorial. Serie ensayos ILPES. Documento 96/34.

Boisier, S. (1998). Teorías y metáforas sobre desarrollo territorial. Revista Austral de Ciencias Sociales, Universidad Austral de Chile, 2, 5-18.

Borja, J. y Castells, M. (1998). Local y global: la gestión de las ciudades en la era de la información. Madrid: Editorial Taurus.

Carvajal, N. (2011). La dinámica del ordenamiento territorial en Colombia: contraste de dinámicas urbano-rurales en la región metropolitana de Bogotá (tesis de doctorado). Universidad de Montreal, Canadá.

Colombia. Senado de la República. (2011). Ley Orgánica de Ordenamiento Territorial. Ley 1454 de 2011. Bogotá D.C.

Departamento Nacional de Planeación (DNP). (2009). Elementos básicos para la planeación y el desarrollo territorial. Bogotá, D.C.

Departamento Nacional de Planeación (DNP). (2010). Orientaciones conceptuales y metodológicas para la formulación de visiones de desarrollo territorial. Bogotá, D.C.

Departamento Nacional de Planeación (DNP). (2011). Visión Boyacá 2019. Bogotá D.C. 174 p.

Fligstein, N. (2001). The Architecture of Markets - An Economic Sociology of Twenty-FirstCentury Capitalist Societies. Princeton: University Press.

Herrera, G. (2009). Análisis fractal de la urbanización de Sogamoso en el periodo 1948-2004. Perspectiva Geográfica, 14, 45-66. Recuperado de http://revistas.uptc. edu.co/revistas/index.php/perspectiva/article/view/1717

Mozas, A. y Bernal, E. (2006). Desarrollo territorial y economía social. CIRIEC-España, Revista de Economía Pública, Social y Cooperativa, 55, 125-140.

Navarro, H. (2005). Transformaciones en los Territorios Periurbanos. En H. Ávila (Coord.), Lo urbano-rural, ¿nuevas expresiones territoriales? (pp. 245275). Cuernavaca, México: UNAM, Centro Regional de Investigaciones Multidisciplinarias. 
Pichierri, A. (2002). Concertation and local development. International Journal of Urban and Regional Research, 26(4), 689-706.

Porras, O. (2006). Desarrollo y ordenamiento territorial para construir un país más competitivo y con regiones competitivas. Revista de la Información Básica (virtual), Centro Andino de Altos Estudios CANDANE. Vol. 1 N. ${ }^{\circ}$ 2. Recuperado de https:// sitios.dane.gov.co/revista_ib/html_r2/articulo5_r2.htm

Programa de Las Naciones Unidas Para el Desarrollo (PNUD). (2002). Serie Desarrollo Humano: Descentralización, organización económica del territorio y potencial de recursos. Cuadernos PNUD. Lima.

Programa de Las Naciones Unidas Para el Desarrollo (PNUD). (2012). Boyacá frente a los objetivos de desarrollo del milenio. Bogotá, D.C.

Sabatini, F. (1990). Planificación del desarrollo regional: desde los sistemas espaciales a los procesos sociales territoriales. Revista Interamericana de Planificación, N. ${ }^{\mathbf{8 9}}$, enero-marzo, pp. 42-75.

Salazar, A. (2010). Transformaciones socio-territoriales en la periferia metropolitana: la ciudad periurbana, estrategias locales y gobernanza en Santiago de Chile. Revista Scripta Nova, 14(331).

Vásquez, B, A. (2009). Desarrollo local, una estrategia para tiempos de crisis. Universitas Forum, 1(2), 11 p. Recuperado de http://hdrnet.org/444/1/barquero_UF2.pdf 